\title{
The Association between Conduct Problems and the Initiation and Progression of Marijuana Use during Adolescence: A Genetic Analysis across Time
}

\author{
Katherine Shelton - Kate Lifford · Tom Fowler • \\ Frances Rice - Mike Neale · Gordon Harold • \\ Anita Thapar $\cdot$ Marianne van den Bree
}

Received: 6 January 2006/ Accepted: 29 June 2006/Published online: 28 November 2006

(C) Springer Science+Business Media, LLC 2006

\begin{abstract}
The present study used a prospective, longitudinal design to investigate genetic and environmental influences on the association between earlier conduct problems and the initiation and progression of marijuana use during adolescence. Parent- and teacher-reported conduct problems assessed at Time 1 (1996) and self-reported marijuana use assessed at Time 2 (2004) were available for 1088 adolescent twin pairs participating in the Cardiff Study of All Wales and North West of England Twins (CaStANET). Using a novel approach to the modeling of initiation and progression dimensions in substance use, findings suggested that the initiation of marijuana use in adolescence was influenced by genetic, common and unique environmental factors. The progression (or frequency) of marijuana use was influenced by genetic and unique environmental factors. Findings for conduct problems indicated that while the presence or absence of conduct problems was largely heritable, the relative severity of conduct problems appeared to be more strongly environmentally influenced. Multivariate model fitting indicated that conduct problems in
\end{abstract}

Edited by Dorret Boomsma

K. Shelton $(\varangle) \cdot$ K. Lifford · T. Fowler .

F. Rice - A. Thapar - M. van den Bree

Department of Psychological Medicine, Cardiff University,

Heath Park, Cardiff CF14 4XN, UK

e-mail: Sheltonkh1@cardiff.ac.uk

M. Neale

Department of Psychiatry and Human Genetics, Virginia

Commonwealth University, Richmond, VA, USA

G. Harold

School of Psychology, Cardiff University, Cardiff, UK childhood and early adolescence made a small but significant contribution to the risk for marijuana use 8 years later.

Keywords Conduct problems - Marijuana use . Adolescence · Initiation · Longitudinal · Genetic . Environmental

\section{Abbreviations \\ CCC Causal common contingency model \\ SDQ Strengths and Difficulties Questionnaire}

Marijuana use can pose a major risk to adolescent health and well-being, yet it remains the most commonly used illicit drug in both the United States and the United Kingdom. In the USA, both availability and rates of marijuana use among adolescents increased sharply during the 1990s, with only a slight decline in recent years (Johnston et al. 2005). In the UK, there was a gradual increase in adolescents reporting marijuana use in the past 12 months, from $10 \%$ in 1998 to $13 \%$ in 2003 , with a slight decline in 2004 to $11 \%$ (National Centre for Social Research 2005). In the United States, $50 \%$ of adolescents reported the use of marijuana by the age of 17 (Johnston et al. 2005) while in the United Kingdom, 38\% of 15-16 year olds had used marijuana (Hibell et al. 2004). Marijuana use is related to a range of deleterious outcomes including lower academic achievement, criminality and mental health problems such as depression and suicidal behaviour (Fergusson et al. 2002; Penning and Barnes 1982; van den Bree and Pickworth 2005). Recent 
research using a twin design has identified conduct problems in childhood and adolescence as a major risk factor for substance use, and marijuana use specifically (e.g. Miles et al. 2002; Silberg et al. 2003; Young et al. 2000).

A series of studies have documented genetic and environmental influences on adolescent marijuana use (e.g. Maes et al. 1999; McGue et al. 2000; Miles et al. 2001). Given the complex processes thought to underlie the link between initiation and progression of drug use however, it is argued that the question should not be whether or not there is a genetic component to drug use per se, but rather what role genetic influences play in the aetiology of drug use over time (Rutter et al. 1999; e.g. Rhee et al. 2003). Recent research has highlighted that the initiation and progression of substances may not be characterised as a single liability of risk (e.g. Agrawal et al. 2005; Heath et al. 1991, 1997; Koopmans et al. 1999). Rather, the initiation and progression phases of substance use may be more appropriately characterised as partially overlapping (or even, independent; Heath et al. 1991). Indeed, while evidence from adult twin studies indicates that there are additive genetic, common environmental and unique environmental factors that are shared between the initiation and progression of marijuana use, there is also evidence of genetic and unique environmental influences that are specific to heavier use of marijuana (Agrawal et al. 2005). The increased influence of genetic factors for heavy substance use possibly reflects the increased involvement of biological processes in chronic use (Kender 2001; van den Bree 2005).

For many individuals, initiation and experimentation with substances occurs during adolescence (Fuller et al. 2005). Adolescents may however, have lower tolerance levels for substances, including marijuana, and become dependent at lower doses than adults (e.g. Chen et al. 1997). Furthermore, adolescent-onset substance misuse is characterized by more rapid development of multiple drug dependencies and more severe psychopathology (e.g. Clark et al. 1998). With this in mind, it is important to consider the relative role of genetic and environmental influences on initiation and progression of substance use in this age group and whether the relationship between these dimensions of use identified with adult samples is also observed in adolescents.

Antisocial behaviour during childhood and adolescence has been linked to substance use in adolescence and early adulthood (e.g. Moffitt et al. 2002; Silberg et al. 2003). Moreover, in the context of other disruptive behaviour disorders, conduct disorder has been shown to be the most predictive of substance use and illicit drug use (e.g. Lynskey and Fergusson 1995; Moss and Lynch 2001). A recent follow-back study of a prospective, longitudinal cohort found that adults with a substance use disorder (alcoholism, marijuana and other drug dependence) were significantly more likely than those without such disorder to have had a conduct and/or oppositional defiant disorder before 15-years old (Kim-Cohen et al. 2003). Strong associations have also been found between conduct disorder symptoms and marijuana use in adolescence for both males and females (Miles et al. 2002). This association was moderately influenced by genetic factors and to a lesser extent, by non-shared environmental factors (see also Young et al. 2000). The findings of a recent longitudinal twin study indicated that conduct problems in adolescence temporally preceded substance use and that the covariation between conduct problems and later substance use was influenced by genetic and common environmental factors (Silberg et al. 2003). These findings mark an important shift away from documenting cross-sectional associations between conduct problems and substance use, from which it is impossible to determine the direction of effects, toward the use of genetically sensitive research designs that assess relationships as they unfold over time. Indeed, in the absence of an experimental design, a primary way in which the nature of the relationship between conduct problems and the initiation and progression of marijuana use during adolescence can begin to be disentangled is to use a prospective, longitudinal twin design.

The present study is among the first to investigate whether the initiation and progression of marijuana use during adolescence reflects a common underlying liability. Extending previous research on the relationship between conduct problems and substance use, this study also examines whether genetic and environmental influences on conduct problems are related to the later initiation of marijuana use in adolescence and in addition, whether conduct problems are implicated in the continued use of marijuana once initiation has occurred. Bivariate genetic analyses were conducted to establish whether marijuana initiation and progression represented a single liability of risk or alternatively, whether these behaviours were more accurately represented as independent liabilities (see Fig. 1). It was hypothesised that genetic, common and unique environmental influences would contribute to the initiation of marijuana use, consistent with the role of social factors in the initial experimentation phase of drug use (Rutter et al. 1999). In accordance with previous studies (Agrawal et al. 2005; Kendler et al. 1999; van den Bree et al. 1998) we hypothesized heavier use (i.e. 


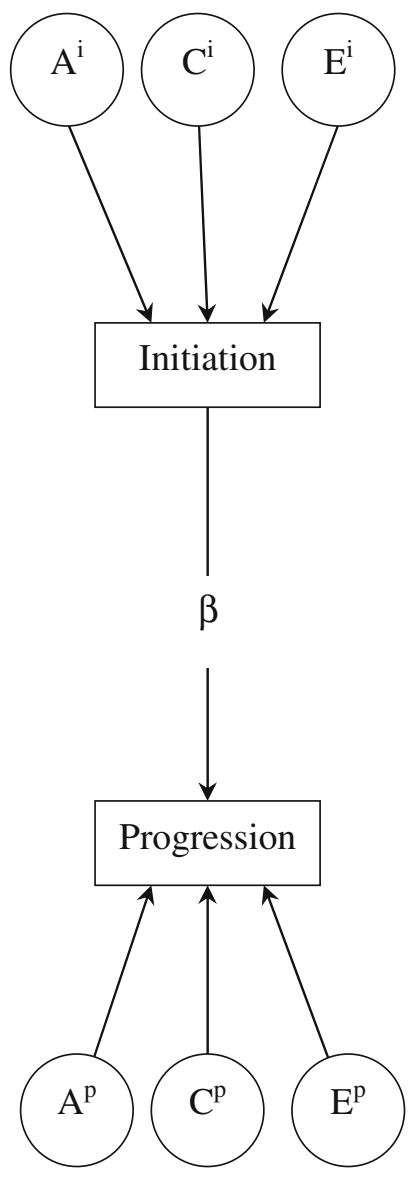

Fig. 1 A conceptual model of the bivariate causal covariance contingency model. Note: Superscript ${ }^{\mathrm{i}}$ refers to factors for initiation. Superscript ${ }^{\mathrm{p}}$ relates to factors specific to progression

the progression of marijuana use, rather than initiation) to be more strongly influenced by genetic factors with a reduced role for common environment. Multivariate analyses assessed the relationship between conduct problems and later marijuana use. It was hypothesised that earlier genetic and environmental influences on conduct problems would be related to the initiation of marijuana use 8 years later. Initiation of marijuana use was expected to remain the strongest predictor of progression of marijuana use after controlling for the influence of conduct problems.

\section{Method}

Sample

The sample used in the present analyses was drawn from the second (1996) and fourth wave (2004) of data collection of the longitudinal Cardiff Study of All Wales and North West of England Twins (CaStANET; Rice et al. 2002; van den Bree et al. in press). The CaStANET register is a population-based twin register, including twins born between 1976 and 1991 in the Cardiff area of South Wales and between 1980 and 1991 for the rest of Wales and the North West of England. This twin register includes families from a systematically ascertained, population-based register of twin births between 1980 and 1991 in Wales and Greater Manchester, UK. Zygosity was assigned using a twin similarity questionnaire completed by parents that is more than $90 \%$ accurate in distinguishing monozygotic (MZ) from dizygotic (DZ) twins and, in a subsample, validated by genotyping DNA markers (Cohen et al. 1975; Nichols and Bilbro 1966; Payton et al. 2001). The CaStANET study received ethical approval from the Multi Centre Research Ethics Committee for Wales, UK.

At the second wave of data collection (1996), questionnaires were mailed to parents of twins aged 5-16 years old. Of 2846 families contacted, 2082 parents returned questionnaires, representing a response rate of $73 \%$ (see Thapar et al. 2000 for a more detailed description of the sample). Parental consent was also obtained to contact the twins' teacher. Of 2168 teachers contacted, 1913 returned questionnaires, representing a response rate of $88 \%$. At the fourth wave of data collection in 2004, questionnaires assessing various aspects of family functioning, parent health and twin psychological adjustment were mailed to families (parents and twins) on the CaStANET twin register with twins aged 11-19 years. Families were sent a reminder postcard, reminder questionnaires and finally, a reminder letter. Non-responding families who might have moved address were traced through General Practitioners. Previous research has shown that adolescent smokers and marijuana users are less likely to respond at follow-up in longitudinal studies (Siddiqui et al. 1996). Extra efforts were therefore made to contact families via telephone where one twin had reported substance use. Of 1755 families with adolescent twins re-contacted in 2004, at least one family member from 1214 families returned questionnaires (1081 parents; 1125 twin pairs, where either or both twins replied) representing a response rate of $69 \%$. Family members who returned questionnaires received a high street store voucher as a token of appreciation for their participation. Demographic statistics indicated that the sample was representative of British families living in the UK region of England and Wales with regard to family constitution, ethnicity, employment and economic factors (Social Trends 2004). 
The present study is based on a subsample of 1088 adolescent twin pairs with information relating to conduct problems (parent and/or teacher report in 1996) and marijuana use in 2004. For clarity, these assessments are referred to as Time 1 (1996) and Time 2 (2004), respectively from this point forward. Of this sample, there was complete information for 895 twin pairs. At Time 1 (1996), the sub-sample of twin pairs were aged 5-13 years (mean $=9.02$ years old; $S D=1.98$, range). At the Time 2 (2004), twin pairs were aged $11-20$ years old (mean $=16.10$ years old; $S D=1.94)$. There were approximately equal numbers of participating boys $(n=960 ; 44.1 \%)$ and girls $(n=1216 ; 55.9 \%)$. The sample comprised 425 monozygotic twins (177 male, 248 female pairs) and 663 dizygotic twins (132 male, 189 female, 342 opposite sex pairs). The majority of twins lived with their biological mother and father $(66.2 \%)$ with smaller proportions living with one biological parent and a stepparent (10.4\%), a single parent (18.7\%) and 'other' (4.6\%). Twins classified as living with 'other' included those who were living with another relative (e.g. grandparents) and twins who lived apart or who were away at university for part of the year. Analyses conducted in which this last group were excluded resulted in a similar pattern of findings to those reported below.

The majority of parent questionnaires (Time 1) were completed by mothers $(93.6 \%)$ with smaller numbers completed by fathers $(5.3 \%)$ and others $(1.1 \%$ e.g. stepparent; grandparent). There was no significant difference in mean levels of conduct problems as a function of the reporter of parent information. Tests indicated that twins who did not respond at the Time 2 follow-up had higher levels of Time 1 conduct problems than twins who did respond $(t=4.809, P<0.001)$. This may indicate a possible bias, whereby adolescents with higher conduct problems who were less likely to respond at follow-up were also those adolescents more likely to use substances, including marijuana.

\section{Measures}

\section{Conduct problems}

Parent and teacher-reported conduct problems were assessed using five items from the Strengths and Difficulties Questionnaire (Goodman 1997; Goodman and Scott 1999). Items included, 'Often tells lies or cheats' 'Steals things', 'Fights or bullies other children', 'Often has temper tantrums or hot tempers' and 'Generally obedient, usually does what adults request' (recoded to reflect disobedient behaviour). Each item was rated, 0 'Doesn't apply', 1 'Applies somewhat' or 2
'Certainly applies'. These items are broadly equivalent to symptoms of conduct disorder as defined by the DSM-IV (American Psychological Association 1994). Parents and teachers reported on behaviour over the last six months. Recent evidence indicates that different informants provide related and unique information about children's antisocial behaviour (Arseneault et al. 2003). Moreover, because children's behaviour can vary between settings, the most valid measurement is that which includes data from more than one informant and from more than one setting or context (Scourfield et al. 2004). Consistent with this argument, conduct problem scores were combined for parent and teacher reports by counting symptoms reported by either parents or teachers as present, thereby capturing each of the five behaviours occurring in both the home and school context. The item-level correlations between parent and teacher reports of conduct problems ranged from $r=0.17$ (stealing) to 0.56 (disobedience), $P<0.001$. The highest rating given by either parent or teacher was taken as the score for that symptom, in other words the occurrence of the behaviour in one context (e.g. the home), was sufficient for it to be counted as present. The internal consistency estimate for the present sample was acceptable $(\alpha=0.76)$ and the five items were added to give a total conduct problems score.

Using criteria outlined by Goodman (1997), conduct problems ranging from 0 to 2 were classified as 'normal', a score of 3-4 was classified as 'borderline' and scores ranging from 5 to 10 were classified as 'abnormal'. Of respondents with conduct information at Time 1, 585 (73\%) monozygotic twins were classified as normal, $154(19 \%)$ as borderline and $67(8 \%)$ as abnormal. Of the dizygotic twins, $877(69 \%)$ were classified as normal, $227(18 \%)$ as borderline and 166 $(13 \%)$ as abnormal. Conduct problems were classified on two dimensions: (1) the presence of conduct problems was indexed as either ' 0 ' for 'normal range' or ' 1 ' for borderline or abnormal conduct problems (2) the second dimension labelled 'borderline/abnormal', distinguished individuals in the borderline and abnormal range for conduct problems. Children in the normal range were coded as missing for borderline/ abnormal problems, borderline conduct problems was coded as ' 0 ' and abnormal conduct problems were coded ' 1 '.

\section{Marijuana use}

The frequency of lifetime marijuana use was assessed using the following item from the Add Health questionnaire (Resnick et al. 1997), 'During your life, how 
many times have you used marijuana?' The six response options ranged from 'Never used marijuana in my life' to 'More than 30 times'. Responses were collapsed to create two binary variables assessing initiation of use and frequency of use. Initiation was indexed as ' 0 ' for never having used marijuana and ' 1 ' for use. Frequency was indexed as light and heavy use with ' 0 ' representing use of marijuana 1-5 times and ' 1 ' representing use of marijuana six or more times. Individuals who had never used marijuana were coded as missing for frequency of use.

\section{Statistical analysis}

The software package Mx (Neale 1997) was used for genetic model fitting. Analyses were conducted using a 'causal common contingent' model which facilitates the expression of marijuana use as a two-stage process incorporating an initiation stage ('upstream', for example whether the individual has ever tried marijuana) that necessarily precedes a progression stage ('downstream', for example, whether the individual uses the marijuana frequently; see Fig. 1; Agrawal et al. 2005; Neale et al. 2006). The model estimates the magnitude of the relationship between initiation and progression by means of a beta pathway between these two stages (see Fig. 1). If the beta coefficient is estimated to be zero, this suggests that the initiation and progression stages for a substance are entirely unrelated processes, i.e. genetic and environmental risk factors for initiation are completely independent from those for progression. Alternatively, if the beta coefficient is estimated to be 1 , this indicates that initiation and progression are entirely overlapping dimensions with identical genetic and environmental risk factors. The $95 \%$ confidence intervals around the beta coefficient provide further information on the degree of overlap between the two stages. Lower limits closer to zero (or below) support independent liabilities and upper limits approaching 1 provide support for identical liabilities. The model also allows the estimation of: (1) additive genetic effects $\left(\mathrm{a}^{2}\right),(2)$ common environmental effects $\left(\mathrm{c}^{2}\right)$, and (3) unique environmental effects $\left(\mathrm{e}^{2}\right)$, on both initiation and progression of substance use.

An important feature of this model is that it is uniquely suited to analysis of data from an adolescent age group, where individuals may have not yet engaged in marijuana use but will go on to become frequent users. The model takes into account the fact that some individuals may be above the liability threshold for progression of marijuana use (i.e. will become frequent users) but because they are not past the age of risk, have not yet initiated marijuana use. As such, their position on the liability distribution of progression is unknown. These individuals are treated as a special case of missing data for progression using the maximum likelihood approach for dealing with missing data in Mx (Neale 1997; Neale et al. 2006). Given the likelihood of an association between age and level of substance use, an age correction was also employed which adjusts the threshold for each twin according to his or her age at the time of questionnaire completion on the distribution of liability to conduct problems and marijuana use. Specifically, the threshold is modeled as a simple linear function:

$t_{i}=t+\operatorname{age}_{i} t_{a}$

where $t$ is the population baseline threshold (for individuals of age zero), $t_{a}$ models the regression of the threshold on age, and age $e_{i}$ is the age in years of the individual $i$ at assessment (Neale et al. 2006).

Models were estimated using full information maximum likelihood (FIML) estimation with raw ordinal data, which included zygosity, twin age, and initiation and progression information relating to conduct problems and/or marijuana use for each twin. The significance of parameters was evaluated using $95 \%$ confidence intervals (CIs), calculated using Mx (Neale and Miller 1997).

\section{Results}

The rate of lifetime marijuana use for the total sample of adolescents was $21.6 \%$. Examining the prevalence of marijuana use for younger and older adolescents highlighted an age-related difference in levels of use. Approximately $11 \%$ of $11-15$ year olds had used marijuana compared with $32.5 \%$ of adolescents aged 16-20 years old. The average age of initiation of marijuana use was 14 years (mean $=14.73$, $S D=1.67$, range: 9-19 years). There was no difference in the prevalence of marijuana use between $\mathrm{MZ}$ $(19.7 \%)$ and DZ twins $\left(22.9 \% ; \chi^{2}=2.987, P=0.084\right)$. Tests indicated greater variance in marijuana use for DZ twins compared to MZ twins $(F=4.220$, $P=0.040)$. A mean difference for a combined estimate of parent and teacher-reported conduct problems between MZ and DZ twins was also found (MZ, mean $=1.68, S D=1.92 ;$ DZ, mean $=1.89, S D=2.13$; $t=2.27, P=0.024)$. Tests revealed greater variation around DZ conduct problems in comparison with $\mathrm{MZ}$ twins $(F=9.938, P=0.002)$. The higher $\mathrm{DZ}$ variances for a parent and teacher rated measure of conduct problems may indicate contrast effects whereby one 
twin is rated as having more behaviour problems than the other twin. On the other hand, the greater variances for DZ twin marijuana use may indicate sibling interaction, for example where one twin is less likely to engage in substance use in response to the other twins' substance using behaviour. However, this is somewhat counterintuitive because research indicates that sibling deviance, and drug and alcohol use specifically, predicts increased substance use (e.g. Stormshak et al. 2004).

Tests of sex differences revealed no sex differences in levels of marijuana initiation or progression (initiation: males, mean $=0.22, S E=0.01$; females, mean $=0.21, S E=0.02 ; t=0.55, P=0.583$; progression: males, mean $=0.32, \quad S E=0.03$; females, mean $=0.41, S E=0.04 ; t=1.75, P=0.081)$ or for the presence and severity of conduct problems (presence: males, mean $=0.29, \quad S E=0.02 ;$ females, mean $=0.31, S E=0.02 ; t=0.78, P=0.437$; severity: males, mean $=0.38, S E=0.03$; females, mean $=0.37$, $S E=0.03 ; t=0.26, P=0.792)$. All figures calculated for pooled twin data were conducted using the survey commands in STATA 9.0 (StataCorp 2005), appropriate for use with twin data when there is non-independence of observations. Preliminary analyses were also conducted to investigate the relationship between a continuously assessed index of conduct problems (1996) and marijuana use (2004). The phenotypic relationship between conduct problems and marijuana use 8 years later was significant $(\beta=0.19, P<0.01$, $R^{2}=0.09$ ). The magnitude of association between conduct problems and marijuana use for dizygotic twins and monozygotic twins was similar, suggesting common environmental influence (MZ twins, $r=0.18$; DZ twins, $r=0.19$ ). Examining the tetrachoric correlations using PRELIS 2.50 (Joreskog and Sorbom 1996) for each of the four constructs (initiation and progression of conduct problems and marijuana use, respectively) suggested genetic and shared environmental influence. The correlations for presence of conduct problems correlations were $r=0.77$ for $\mathrm{MZ}$ twins and 0.46 for DZ twins indicating genetic and environmental influence, while the correlations for the borderline/abnormal conduct problems construct were $r=0.21$ for $\mathrm{MZ}$ twins and 0.30 for $\mathrm{DZ}$ twins, indicating stronger environmental influence. The results for initiation of marijuana use indicated genetic and shared environmental effects (MZ $r=0.80 ; \mathrm{DZ}$ $r=0.70$ ), while progression of marijuana use appeared to be more strongly genetically influenced (MZ $r=0.68$; DZ $r=0.13$ ).

The relationship between the liability to initiation and progression of conduct problems and marijuana use

The results of model tests for conduct problems and marijuana use, respectively, are presented in Table 1.

\section{Conduct problems}

To facilitate later multivariate tests of the relationship between earlier conduct problems (Time 1) and later marijuana use (Time 2), a CCC model was estimated for conduct problems. Given the differences found between $\mathrm{MZ}$ and $\mathrm{DZ}$ twins in levels of conduct problems, model tests were conducted in which thresholds for the different twin groups were allowed to vary. This provided a better fit to the data than a model in which thresholds were estimated to be the same between twin groups and these results are reported $\left(\chi^{2}=7.853, d f=2, P<0.05\right)$. Substantively however, the pattern of results was the same. The liability to any conduct problems accounted for a large proportion, but not all, of the variance in the liability to be classified as borderline/abnormal for conduct problems $(\beta=0.74$; $\mathrm{CI}=0.22,0.95)$. The presence of conduct problems was mainly influenced by genetic $(69 \%)$ and non-shared environmental factors (22\%). Borderline/abnormal conduct problems were influenced by common environmental (27\%) and non-shared environmental (73\%) factors. The threshold estimates were 0.47 for presence

Table 1 CCC model tests for conduct problems and marijuana use

\begin{tabular}{|c|c|c|c|c|c|c|c|}
\hline & \multicolumn{3}{|l|}{ Initiation } & \multirow[b]{2}{*}{$\beta$} & \multicolumn{3}{|l|}{ Progression } \\
\hline & $a^{2}$ & $c^{2}$ & $\mathrm{e}^{2}$ & & $\overline{a^{2}}$ & $c^{2}$ & $e^{2}$ \\
\hline \multicolumn{8}{|l|}{ Time 1} \\
\hline Conduct problems & $\begin{array}{l}0.69 \\
\quad(0.41,0.86)\end{array}$ & $\begin{array}{l}0.08 \\
\quad(0.02,0.32)\end{array}$ & $\begin{array}{l}0.22 \\
\quad(0.14,0.32)\end{array}$ & $\begin{array}{l}0.74 \\
\quad(0.22,0.95)\end{array}$ & $\begin{array}{l}0.00 \\
\quad(0.00,0.00)\end{array}$ & $\begin{array}{l}0.27 \\
\quad(0.00,0.37)\end{array}$ & $\begin{array}{l}0.73 \\
\quad(0.05,0.76)\end{array}$ \\
\hline \multicolumn{8}{|l|}{ Time 2} \\
\hline Marijuana use & $\begin{array}{l}0.35 \\
\quad(0.05,0.63)\end{array}$ & $\begin{array}{l}0.47 \\
\quad(0.24,0.71)\end{array}$ & $\begin{array}{l}0.18 \\
\quad(0.10,0.36)\end{array}$ & $\begin{array}{l}0.88 \\
\quad(0.38,0.99)\end{array}$ & $\begin{array}{l}0.64 \\
\quad(0.00,0.65)\end{array}$ & $\begin{array}{l}0.00 \\
\quad(0.00,0.00)\end{array}$ & $\begin{array}{l}0.36 \\
\quad(0.00,0.48)\end{array}$ \\
\hline
\end{tabular}


and 1.10 for severity. A comparison of goodness-of-fit indices indicated that modelling thresholds as a function of age did not significantly improve the fit of the model $\left(\chi^{2}=4.302, d f=2, P>0.10\right)$.

\section{Marijuana use}

The results for marijuana use indicated that the initiation of use was explained by a heritable component $(35 \%)$, common environment $(47 \%)$ and nonshared environment $(18 \%)$. In contrast, the frequency of marijuana use was explained by a heritable component $(64 \%)$ and a non-shared environment component $(36 \%)$. The threshold estimates were 0.57 for initiation and 1.17 for frequency. The beta value $(\beta=0.88$; $\mathrm{CI}=0.38,0.99)$ represents the genetic and environmental influences on initiation that are, in turn, transmitted to the frequency of marijuana use. This value, which is less than unity, indicated that while the liabilities for the initiation and frequency of marijuana use were not independent, neither could these dimensions be assumed to reflect a single liability of risk. In other words, the liability to initiate use of marijuana accounted for a substantial proportion (approximately $77 \%$ ), but not all, of the variance in the liability to more frequent use of marijuana. The CI's around beta (0.38-0.99) further indicated that the two liabilities were moderately to strongly related but not identical. Goodness-of-fit statistics indicated that a model in which age-corrected thresholds were estimated provided a better fit to the data than a model that did not include age-corrected estimates $\left(\chi^{2}=56.155\right.$, $d f=2, P<0.001$ ), corroborating our findings of a higher prevalence of increased marijuana use in older adolescents.

\section{Multivariate analyses}

Analyses were conducted to examine whether the liability to conduct problems during childhood and early adolescence was a risk factor for the liability to initiate and use marijuana 8 years later. The model was again estimated using full information maximum likelihood (FIML) estimation with raw ordinal data, which included zygosity, twin age, and 'initiation' and 'progression' information relating to conduct problems and marijuana use for each twin. In addition to paths estimated from each initiation variable to the progression variable, paths were estimated from presence of and borderline/abnormal conduct problems at Time 1 to marijuana initiation and progression at Time 2 . Figure 2 presents the results for the full model, in which all six pathways between conduct problems (Time 1) and marijuana use (Time 2) were estimated. This model provided similar estimates of the relationship between each classification of conduct problems and the initiation and frequency of marijuana use, respectively. Minor fluctuations were found between models (bivariate model to the full multivariate model) in the genetic and environmental estimates for conduct problems, which can be expected to be a result of the model estimation. Fixing estimates in the full model to their bivariate values would not change the substantive interpretation of the multivariate results, nor would it yield a significant difference in fit. However, the multivariate results should generally be regarded as superior, because they use more information.

The strongest beta-paths were observed within traits, between initiation and progression variables for both conduct problems and marijuana use. No significant effects were observed between conduct problems and later marijuana use $(\beta$ range $=0.04$ $0.17)$. In the context of strong effects from initiation to frequency of marijuana use $(\beta=0.75)$, associations between conduct problems and the frequency of marijuana use were relatively weak. As the bivariate analysis described above suggested however, there were also relatively high levels of covariation in the liabilities for presence and borderline/abnormal conduct problems. This may have affected the power of either variable to predict initiation of marijuana use. To investigate this possibility, nested models were analysed to test the effect of dropping pathways between initiation and progression constructs and between conduct problems and marijuana use. Models were fitted whereby the paths between liability to conduct problems, severity of conduct problems, liability to marijuana initiation and frequency of use were dropped in turn and the deterioration in chisquare fit compared to a model in which all possible path coefficients from Time 1 conduct problems to Time 2 marijuana use were estimated (see Table 2 for model fitting results). These results showed that when the path from liability to conduct problems and severity of conduct problems was dropped there was a significant reduction in model fit, compared to a full model. This was also the case when the path from liability to marijuana initiation and frequency of use was dropped. When the path from borderline/abnormal conduct problems to initiation of marijuana use was dropped, the pathway from presence of conduct problems to the initiation of marijuana use became statistically significant $(\beta=0.23, \quad \mathrm{CI}=0.13,0.33)$. Likewise, when the path from presence of conduct 
Fig. 2 A causal covariance contingency model of the longitudinal relationship between conduct problems and marijuana use. Note: Superscript ${ }^{\mathrm{i}}$ refers to factors for initiation. Superscript ${ }^{p}$ relates to factors specific to progression. 95\% confidence intervals appear in brackets
Time 1 (1996)
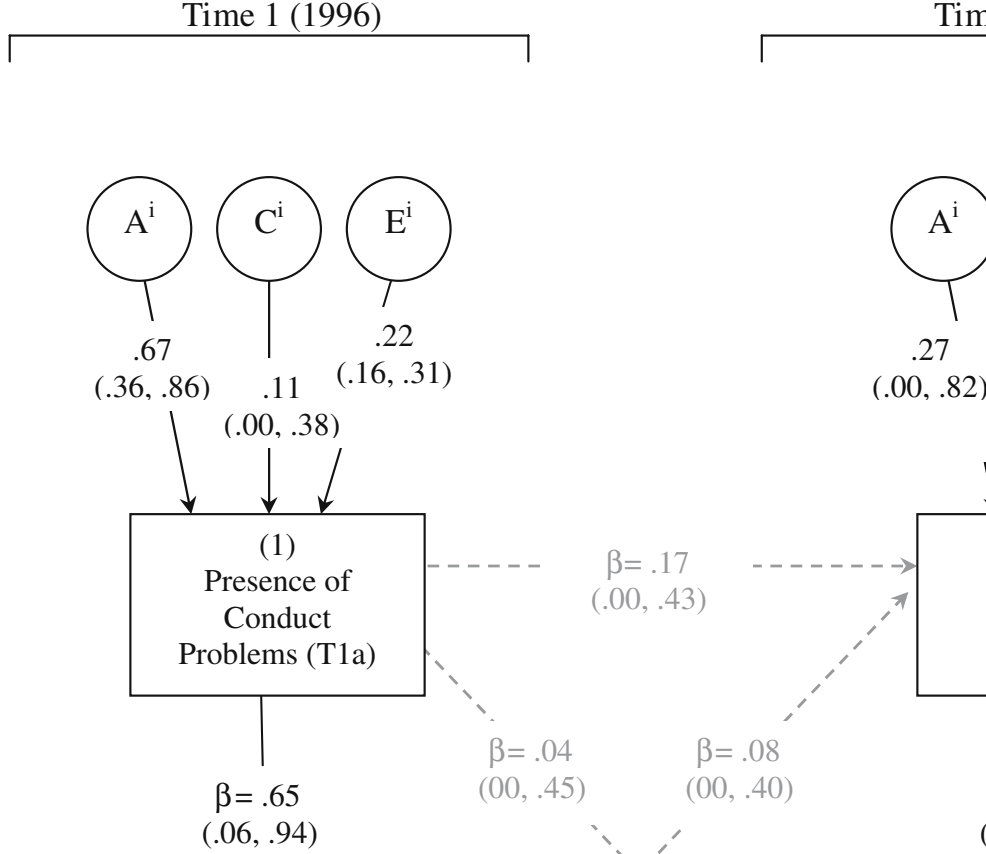

.27

$(.00, .82)$
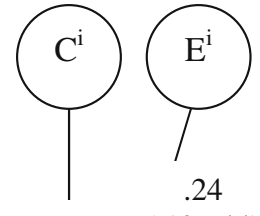

.24

$(.13, .44)$

$(.15, .77)$

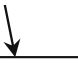

(3)

Initiation of

Marijuana

Use (T2a)

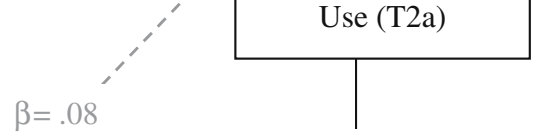

$(00, .40) \quad \beta=.75$

$(.12, .90)$

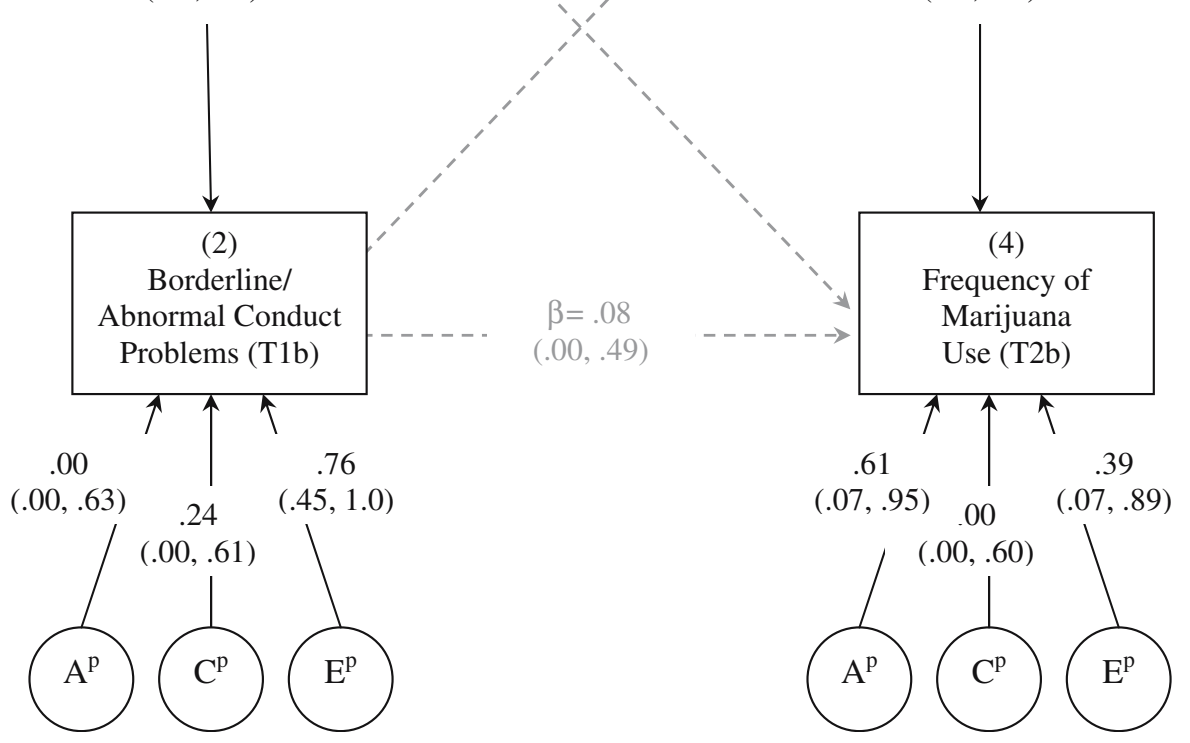

Table 2 Multivariate model fitting for relations between conduct problems and marijuana use

\begin{tabular}{lllll}
\hline Estimated model & $\begin{array}{l}\text { Beta } \\
\text { coefficient } \\
\text { set at zero }\end{array}$ & -2LL & $d f$ & $\chi^{2}$ \\
\hline $\begin{array}{l}\text { Model 1-Full } \\
\text { theoretical }\end{array}$ & - & 5433.945 & 5124 & - \\
$\quad$ model & & & & \\
Model 2 & $\beta 21$ & 5438.720 & 5125 & $4.78^{*}$ \\
Model 3 & $\beta 31$ & 5436.969 & 5125 & 3.02 \\
Model 4 & $\beta 41$ & 5433.847 & 5125 & 0.10 \\
Model 5 & $\beta 32$ & 5433.771 & 5125 & 0.17 \\
Model 6 & $\beta 42$ & 5433.825 & 5125 & 0.12 \\
Model 7 & $\beta 43$ & 5442.258 & 5125 & $8.31^{* *}$ \\
\hline
\end{tabular}

Note: $* P<0.05, * * P<0.01$ problems to initiation of marijuana use was dropped, the pathway from borderline/abnormal conduct problems to the initiation of marijuana use also became statistically significant $(\beta=0.24, \quad \mathrm{CI}=0.22,0.35)$. Inspecting the deterioration in model fit when each of these two paths was dropped indicated that when the pathway from presence of conduct problems to the initiation of marijuana use was dropped, there was a trend toward a significant reduction in model fit, $\left(\chi^{2}=3.02, d f=1, P<0.10\right)$. In contrast, when the pathway from borderline/abnormal conduct problems to the initiation of marijuana use was dropped, a significant drop in model fit was not observed $\left(\chi^{2}=0.17, d f=1, P>0.10\right)$. 


\section{Discussion}

This study is among the first to investigate the relationship between the initiation and progression dimensions of substance use with an adolescent twin sample and highlights that the initiation and progression of marijuana use, although strongly related, cannot be assumed to have a single liability of risk. The findings of this study also provide insight into the relationship between conduct problems and adolescent substance use. Specifically, the results indicate that in a UK sample, the longitudinal association between the liability to conduct problems during childhood and early adolescence and the liability to initiate and progress marijuana use in adolescence and early adulthood are not strongly related. Thus, other risk factors and the mechanisms through which they exert effects on the initiation and progression of marijuana use in adolescence should also be investigated.

Consistent with previous research examining the relationship between initiation and progression (or use and abuse) of substances in adult populations, additive genetic, common and non-shared environmental influences were found for initiation of marijuana while factors specific to progression were influenced by genetic and non-shared environment with no role for common environment (e.g. Agrawal et al. 2005; Heath et al. 1997; Kendler and Prescott 1998; Koopmans et al. 1999). These findings concur with the view that genetically influenced biological mechanisms appear to play a more important role in the aetiology of problem use and substance dependence (Kendler et al. 1999; van den Bree 2005). Interestingly, when conduct problems were introduced as predictors of marijuana initiation and progression in the multivariate model, there was a slight reduction in the genetic estimate and an increase in the environmental influence. This was particularly the case for initiation of marijuana use. It is also interesting to note that while not independent, the initiation and frequency of marijuana use, to some degree, represent different liabilities of risk. Conceptually, this indicates that the use of marijuana by adolescents does not always and inevitably lead to more frequent use and that there may be different risk factors underlying initiation and progression to more frequent marijuana use. Studies into risk factors that predispose individuals to continued use of marijuana after first experimentation with the drug will be helpful in increasing insight into which adolescents are at greatest risk for problem use. For example, van den Bree and Pickworth (2005) found that some risk factors predict both initiation of experimental marijuana use and progression to regular use, while others are specific to each stage.

The results for conduct problems indicated that while the presence or absence of conduct problems were largely heritable, the relative severity of conduct problems, categorised as 'borderline' and 'abnormal' appeared to be more strongly environmentally influenced. However, we cannot rule out the possibility of greater measurement error at the upper end of the distribution contributing to a large non-shared environment estimate. The beta coefficient between the two dimensions suggested that they mostly, but not entirely overlap ( $\beta=0.74$; see Table 1 ), with wide $95 \%$ CIs $(0.22,0.95)$. Conceptually and epidemiologically, recent evidence (e.g. Pickles et al. 2001; van den Oord et al. 2003) indicates that conduct problems may be best regarded as a single liability of risk where there is no demarcation between normality and psychopathology (Rutter 2003). The categorization of conduct problems into dichotomous variables may have affected the power to predict later marijuana use. Preliminary analyses indicated that conduct problems assessed as a continuous variable predicted later marijuana use, although the strength of association was not large $(\beta=0.19 ; P<0.01)$. Likewise, model tests in which presence and severity of conduct problems were assessed separately indicated small but significant effects from conduct problems to the initiation of marijuana use. Although the trend toward a significant drop in model fit when the path from severity of conduct problems to initiation of marijuana use was removed suggests that it is the presence rather than severity of conduct problems that is important in the prediction of marijuana use initiation, this is speculative and should be replicated in independent samples of larger number. Moreover, it will be interesting in the future (as these models become available) to repeat these analyses including conduct problems as a dimensional measure with a single liability. The findings of the present study also suggest that this modelling technique could be applied to questions relating to the transition from symptoms to diagnosis, particularly when longitudinal data are available to examine this relationship over time (see Neale et al. 2006).

A limitation of the present study was that the results of both the bivariate and multivariate model tests had wide confidence intervals around the progression variables (borderline/abnormal conduct problems and frequency of marijuana use). These possibly reflect the low frequencies of adolescents with high levels of conduct problems and who had initiated marijuana use. 
For instance, preliminary analyses indicated that adolescents who did not respond at Time 2 (2004) had higher levels of conduct problems at Time 1 (1996). Thus, the present analyses may represent a conservative estimate of the influence of conduct problems on later initiation and progression of marijuana use. In addition, heterogeneity in the relationship between conduct problems and marijuana use as a function of the wide age range of the sample, together with a reliance on a single item to index marijuana use, may have affected the power to detect an association. In addition, in light of the strong relations between the initiation and progression variables, the cross-trait paths are relatively small and larger sample sizes than we currently have available are needed to obtain more conclusive nested model fitting results for the crosstrait paths. Finally, it should be noted that while the present study provides insights regarding the phenotypic relationship between conduct problems and the initiation and progression of marijuana use in adolescence together with sources of genetic and environmental influence on each index of conduct problems and marijuana use, it does not assess genetic and environmental sources of covariance between these behaviours (see Neale et al. 2006). In addition to addressing the caveats outlined above therefore, an important direction for further research is to assess these influences.

Previous research found sex differences in the prevalence of marijuana use (greater use among males; Johnston et al. 2005) and in the genetic and environmental influences on adolescent male and female marijuana initiation (e.g. Rhee et al. 2003). Recent research has also identified sex differences in the pattern of relations between the severity of conduct problems and the later initiation of marijuana use (Pedersen et al. 2001). The binary nature of the study variables together with the sample size did not permit model tests incorporating sex differences. Nevertheless, an important direction for future research is to test causal common contingent models for sex differences in the pattern of relations between conduct problems and marijuana use in an adolescent age group.

Notwithstanding these limitations, the present study extends previous research by investigating longitudinal relationships between conduct problems and marijuana use during adolescence using a modelling approach that enabled age appropriate adjustments to estimates of the relationship between the initiation and progression dimensions of substance use. The findings are also consistent with previous research indicating stronger associations between conduct problems and substance use within than across-time (e.g. Miles et al. 2002;
Silberg et al. 2003). As such, they suggest that interventions aimed at helping children with conduct problems in the years preceding the onset of substance use are unlikely to reduce the risk of marijuana use in adolescence and young adulthood. Other risk factors need to be investigated. An important direction for future research will be to develop models that can test these processes with greater precision. For example, models are required that permit the testing of risk factors assessed using continuous measures as predictors of initiation and progression of substance use and the investigation of moderators of the liability to initiate and progress in substance use when these reflect independent liabilities of risk. The findings of this prospective, longitudinal study represent a first step in examining the risk factors that influence the initiation of substance use and the progression to more frequent use during adolescence and young adulthood.

Acknowledgments We would like to thank the families who participated in the Cardiff Study of All Wales and North West of England Twins for their time and contribution to this project. Ivan Nikolov from the Biostatistics Bioinformatics Unit, Department of Psychological Medicine at Cardiff University provided database management and support. Kate Lifford was funded by the Biostatistics Bioinformatics Unit, Department of Psychological Medicine. The development of this manuscript was supported by grants awarded by the Wellcome Trust (GR073063) to Marianne van den Bree, Anita Thapar, Michael C. Neale, Gordon Harold and Jane Scourfield and by the European Research Advisory Board awarded to Marianne van den Bree, Anita Thapar and Jane Scourfield. Support was also provided by an NIH grant (DA018673) awarded to Michael C. Neale.

\section{References}

American Psychological Association (1994) Diagnostic criteria from DSM-IV-TR

Agrawal A, Neale MC, Jacobson KC, Prescott CA, Kendler KA (2005) Illicit drug use and abuse/dependence: modeling of two-stage variables using the CCC approach. Addict Behav 30:1043-1048

Arsenault L, Moffitt TE, Caspi A, Taylor A, Rijsdijk FV, Jaffee SR, Ablow JC, Measelle JR (2003) Strong genetic effects on cross-situational antisocial behaviour among 5-year-old children according to mothers, teachers, observers, and twins' self-reports. J Child Psychol Psychiatry 44:832-848

Chen K, Kandel DB, Davies M (1997) Relationships between frequency and quantity of marijuana use and last year proxy dependence among adolescents and adults in the United States. Drug Alcohol Dependence 46:53-67

Clark DB, Neighbors BD, Lesnick LA, Lynch KG, Donovan JE (1998) Family functioning and adolescent alcohol use disorders. J FamilyPsychol 12:81-92

Cohen D, Dibble E, Grawe JM, Pollin W (1975) Reliably separating identical twins from fraternal twins. Arch Gen Psychiatry 32:1371-1375

Fergusson DM, Horwood LJ, Swain-Campbell N (2002) Cannabis use and psychosocial adjustment in adolescence and young adulthood. Addiction 97:1123-1135 
Fuller E (Editor), Bates B, Blenkinsop S, Boreham R, Hills A, McGee A et al (2005) Smoking, drinking and drug use among young people in England in 2004. Health and Social Care Information Centre, Leeds

Goodman R (1997) The strengths and difficulties questionnaire: a research note. J Child Psychol Psychiatry 38:581-586

Goodman R, Scott S (1999) Comparing the strengths and difficulties questionnaire and the child behavior checklist: is small beautiful? J Abnorm Child Psychol 27:17-24

Heath AC, Bucholz KK, Madden PAF, Dinwiddie SH, Slutske WS, Bierut LJ, Statham DJ, Dunne MP, Whitfield JB, Martin NG (1997) Genetic and environmental contributions to alcohol dependence risk in a national twin sample: consistency of findings in women and men. Psychol Med 27:1381-1396

Heath AC, Meyer J, Eaves LJ, Martin NG (1991) The inheritance of alcohol consumption patterns in a general population twin sample: I. Multidimensional scaling of quantity/ frequency data. J Studies Alcohol 52:345-352

Hibell B, Andersson B, Bjarnason T, Ahlström S, Balakireva O, Kokkevi A, Morgan M (2004) The ESPAD Report 2003. Alcohol and other drug use among students in 35 European countries. The Swedish Council for Information on Alcohol and Other Drugs (CAN) and the Pompidou Group at the Council of Europe. Stockholm, Sweden. 436 pp

Johnston LD, O'Malley PM, Bachman JG, Schulenberg JE (2005) Monitoring the future national results on adolescent drug use: overview of key findings, 2004 (NIH Publication No. 05-5726). National Institute on Drug Abuse, Bethesda, $\mathrm{MD}, 66 \mathrm{pp}$

Jöreskog KG, Sörbom D (1996) Prelis 2.50. Scientific Software International, Inc

Kender KS (2001) Twin studies in psychiatric illness: an update. Arch Gen Psychiatry 58:1005-1014

Kendler KS, Neale MC, Sullivan P, Corey LA, Gardner CO, Prescott CA (1999) A population-based twin study in women of smoking initiation and nicotine dependence. Psychol Med 29:299-308

Kendler KS, Prescott CA (1998) Cannabis use, abuse, and dependence in a population-based sample of female twins. Arch Gen Psychiatry 155: 1016-1022

Kim-Cohen J, Caspi A, Moffitt TE, Harrington HL, Milne BJ, Poulton R (2003) Prior juvenile diagnoses in adults with mental disorder: developmental follow-back of a prospective-longitudinal cohort. Arch Gen Psychiatry 60:709-717

Koopmans JR, Slutske WS, Heath AC, Neale MC (1999) The genetics of smoking initiation and quantity smoked in Dutch adolescent and young adult twins. Behav Genet 29:383-393

Lynskey MT, Fergusson DM (1995) Childhood conduct problems, attention deficit behaviours, and adolescent alcohol, tobacco, and illicit drug use. J Abnorm Child Psychol 23:281-302

Maes HH, Woodard CE, Murrelle L, Meyer JM, Silberg JL, Hewitt JK, Rutter M, Simonoff E, Pickles A, Carbonneau R, Neale MC, Eaves LJ (1999) Tobacco, alcohol, and drug use in 8-16 year old twins: the Virginia twin study of adolescent behavioral development. J Studies Alcohol 60:293-305

McGue M, Elkins I, Iacono WG (2000) Genetic and environmental influences on adolescent substance use and abuse. Am J Med Genet 96:671-677

Miles DR, van den Bree MBM, Gupman AE, Newlin DB, Glantz MD, Pickens RW (2001) A twin study on sensation seeking, risk taking behaviour and marijuana use. Drug Alcohol Dependence 62:57-68
Miles DR, van den Bree MBM, Pickens RW (2002) Sex differences in shared genetic and environmental influences between conduct disorder symptoms and marijuana use in adolescents. Am J Med Genet 114:159-168

Moffitt TE, Caspi A, Harrington H, Milne BJ (2002) Males on the life-course persistent and adolescence-limited anti-social pathways: follow-up at age 26. Devel Psychopathol 14:179207

Moss HB, Lynch KG (2001) Comorbid disruptive behaviour disorder symptoms and their relationship to adolescent alcohol use disorders. Drug Alcohol Dependence 64:75-83

National Centre for Social Research (2005) Smoking, drinking and drug use among young people in England in 2004. Retrieved 20th August, 2005, from http://www.nfer.ac.uk/ index.cfm

Neale MC (1997) Mx: statistical modeling, 4th edn. Department of Psychiatry, Virginia Commonwealth University, Richmond, VA

Neale MC, Harvey E, Maes HHM, Sullivan PF, Kendler KS (2006) Extensions to the modeling of initiation and progression. Behav Genet 36:507-524

Neale MC, Miller MB (1997) The use of likelihood-based confidence intervals in genetic models. Behav Genet 27:113120

Nichols RC, Bilbro WC Jr (1966) The diagnoses of twin zygosity. Acta Genet Stat Med 16:265-275

Payton A, Holmes J, Barrett JH, Sham P, Harrington R, McGuffin P, Owen M, Ollier W, Worthington J, Thapar A (2001) Susceptibility genes for a trait measure of attention deficit hyperactivity disorder: a pilot study in a non-clinical sample of twins. Psychiatry Res 105:273-278

Pedersen W, Mastekaasa A, Wichstrøm L (2001) Conduct problems and early cannabis initiation: a longitudinal study of gender differences. Addiction 96:415-431

Penning M, Barnes GE (1982) Adolescent marijuana use: a review. Int J Addiction 17:749-791

Pickles A, Rowe R, Simonoff E, Foley D, Rutter M, Silberg J (2001) Child psychiatric symptoms and psychosocial impairment: relationship and prognostic significance. Br J Psychiatry 179:230-235

Resnick MD, Bearman PS, Blum RW, Bauman KE, Harris KM, Jones J, Tabor J, Beuhring T, Sieving RE, Shew M, Ireland M, Bearinger LH, Udry JR (1997) Protecting adolescents from harm. Findings from the National Longitudinal Study on Adolescent Health. JAMA 278:823-832

Rhee HR, Hewitt JK, Young SE, Corley RP, Crowley TJ, Stallings MC (2003) Genetic and environmental influences on substance initiation, use, and problem use in adolescents. Arch Gen Psychiatry 60:1256-1264

Rice F, Harold GT, Thapar A (2002) Assessing the effects of age, sex and shared environment on the genetic aetiology of depression in childhood and adolescence. J Child Psychol Psychiatry 43:1039-1051

Rutter M (2003) Categories, dimensions, and the mental health of children and adolescents. In King JA, Ferris CF, Lederhendler II (eds), Annals of the New York Academy of Sciences, vol 1008, pp 11-21

Rutter M, Silberg J, O'Connor T, Simonoff E (1999) Genetics and child psychiatry: II empirical research findings. J Child Psychol Psychiatry 40:19-55

Scourfield J, van den Bree MBM, Martin N, McGuffin P (2004) Conduct problems in children and adolescents. Arch Gen Psychiatry 61:489-496

Siddiqui O, Flay BR, Hu FB (1996) Factors affecting attrition in a longitudinal smoking prevention study. Preventive Med 25:554-560 
Silberg J, Rutter M, D’Onofrio B, Eaves L (2003) Genetic and environmental risk factors in adolescent substance use. $\mathrm{J}$ Child Psychol Psychiatry 44:664-676

Social Trends (2004) Office of National Statistics, London

Statacorp (2005) Stata Statistical Software: Release 9. Statcorp LP, College Station, TX

Stormshak EA, Comeau CA, Shepard SA (2004) The relative contribution of sibling deviance and peer deviance in the prediction of substance use across middle childhood. J Abnormal Child Psychol 32:635-649

Thapar A, Ross K, Harrington R et al (2000) Does the definition of ADHD affect heritability? J Am Acad Child Adolesc Psychiatry 39:1528-1536

van den Bree MBM (2005) Combining research approaches to advance our understanding of drug addiction. Curr Psychiatry Rep 7:1-8

van den Bree MBM, Pickworth WB (2005) Risk factors predicting changes in marijuana involvement in teenagers. Arch Gen Psychiatry 62:311-319 van den Bree MBM, Johnson EO, Neale MC, Pickens RW (1998) Genetic and environmental influences on drug use and abuse/dependence in male and female twins. Drug Alcohol Dependence 52:231-241

van den Bree M, Rice F, Fowler T, Shelton K, Lifford K, Scourfield J, Harold G, Thapar A. The Cardiff Study of All Wales and North West of England Twins (CaStANET): A longitudinal research programme of child and adolescent development. Twin Res Hum Genet (in press)

van den Oord EJCG, Pickles A, Waldman ID (2003) Normal variation and abnormality: an empirical study of the liability distributions underlying depression and delinquency. J Child Psychol Psychiatry 44:180-192

Young SE, Stallings MC, Corley RP, Krauter KS, Hewitt JK (2000) Genetic and environmental influences on behavioural disinhibition. Am J Med Genet 96:684-695 